\title{
Hashimoto's Encephalopathy with Reversible Diffuse Leukoencephalopathy
}

\author{
Woosub Hwang, $\mathrm{MD}^{1}$, Da-Eun Jeong, $\mathrm{MD}^{2}$ \\ ${ }^{1}$ Department of Neurology, Daegu Fatima Hospital, Daegu; ${ }^{2}$ Department of Neurology, Yeungnam University College of Medicine, Daegu, Korea
}

Background: The magnetic resonance imaging (MRI) findings of Hashimoto's encephalopathy (HE) are variable; images range from having a normal appearance, to showing ischemic lesions, demyelination, or vasogenic edema.

Case Report: A 57-year-old woman presented with a sudden onset of dysarthria and dizziness. Routine blood tests were normal and the MRI was unremarkable. After admission, she developed aphasic seizures and myoclonus, and eventually became comatose. A follow up MRI showed diffuse high signal intensities at the bilateral cerebral white matter on fluid attenuated inversion recovery imaging. The serum titers of the anti-thyroglobulin antibody and anti-thyroperoxidase antibody were increased. After 5 days of 1,000 mg/day of intravenous methylprednisolone infusion, she recovered rapidly.

Conclusion: We propose that repeated brain MRIs are necessary for the diagnosis of HE. Diffuse leukoencephalopathy may be a pathologic finding on MRI and, in this case study, was shown to be reversible after applying an appropriate treatment.

J Neurocrit Care 2018;11(1):54-57

Key words: Hashimoto's disease; Leukoencephalopathies; Magnetic resonance imaging

\author{
Received May 24, 2018 \\ Revised June 6, 2018 \\ Accepted June 7, 2018 \\ Corresponding Author: \\ Da-Eun Jeong, MD \\ Department of Neurology, Yeungnam \\ University College of Medicine, 170 \\ Hyeonchung-ro Nam-gu, Daegu 42415, \\ Korea \\ Tel: +82-53-620-3684 \\ Fax: +82-53-627-1688 \\ E-mail: doctorjung86@gmail.com
}

Copyright $\odot 2018$ The Korean Neurocritical Care Society

\section{INTRODUCTION}

Hashimoto's encephalopathy (HE) is an uncommon autoimmune disease involving the central nervous system and is characterized by high titers of antithyroid antibodies and various neuropsychological symptoms. ${ }^{1}$ The clinical manifestations of $\mathrm{HE}$ are diverse, and may include minor cognitive impairment, tremors, myoclonus, status epilepticus, and coma. ${ }^{2}$ The magnetic resonance imaging (MRI) findings of $\mathrm{HE}$ are variable, images range from having a normal appearance, to showing ischemic lesions, demyelination, or vasogenic edema. ${ }^{3}$ Herein we report a case of
HE with reversible diffuse leukoencephalopathy on MRI.

\section{CASE REPORT}

A 57-year-old woman presented with sudden onset of transient dysarthria and dizziness. She underwent a mastectomy 10 years ago for breast cancer. She was healthy until this presentation and took no medication. A neurologic examination revealed dysarthria and mild postural tremor on both arms. Routine blood tests were within normal limits. Electrocardiography (EEG) showed no abnormalities. Both

(cc) This is an Open Access article distributed under the terms of the Creative Commons Attribution Non-Commercial License (http://creativecommons.org/licenses/by$\mathrm{nc} / 4.0$ ) which permits unrestricted non-commercial use, distribution, and reproduction in any medium, provided the original work is properly cited. 
the brain MRI and MR angiography were unremarkable. The patient was diagnosis with transient ischemia attack and received aspirin and rosuvastatin. The initial thyroid function test was normal as T3 84.50 ng/dL (normal, 80-200 ng/dL), free T4 $1.17 \mathrm{ng} / \mathrm{dL}$ (normal, 0.93-1.70 ng/dL), thyroid stimulating hormone (TSH) $8.53 \mu \mathrm{lU} / \mathrm{mL}$ (normal, 0.5-8.9 $\mu \mathrm{lU} / \mathrm{mL}$ ). Four days after admission, her speech became slower and she took more than 10 seconds to answer a simple question. However, the follow-up diffusion weighted imaging (DWI) was normal. We changed the medication from aspirin to cilostazol and clopidogrel. The EEG was repeated and showed bifrontal dominant slow waves and frequent sharp waves on the $\mathrm{F}_{3} / \mathrm{F}_{4}$ electrodes. We administered oxcarbazepine $450 \mathrm{mg} /$ day and the antiplatelet agents and statins were withdrawn. On day 8 , she developed intermittent aphasia and then had periods of brief confusion several times a day. The results of the language function test that was performed when aphasia occurred are as fol- lows: naming was 0 of 6 , fluency was 1 of 4 , repetition was 1 of 4, comprehension was 1 of 4 . We considered she had global aphasia and postictal confusion due to a complex partial seizure, i.e., ictal aphasia. We added levetiracetam 1,000 mg/day and phenytoin 300 mg/day. Eleven days after admission, her mental status had worsened; she was in a coma with intermittent whole-body myoclonus. On the follow-up EEG, there was an ictal discharge that started from the bifrontal area as a semi-rhythmic theta to delta wave at 4-6 Hz. We administered valproate $900 \mathrm{mg} /$ day. On day 13, the follow-up MRI showed diffuse confluent high signal intensities on the bilateral cerebral white matter on DWI (A), apparent diffusion coefficient (ADC) mapping (B) and fluid-attenuated inversion recovery (FLAIR) images (C). We considered that the subtle high signal intensity on DWI was due to the T2 shiny effect, and the MRI findings were compatible with vasogenic edema (Fig. 1). Cerebrospinal fluid analysis revealed a slightly increased
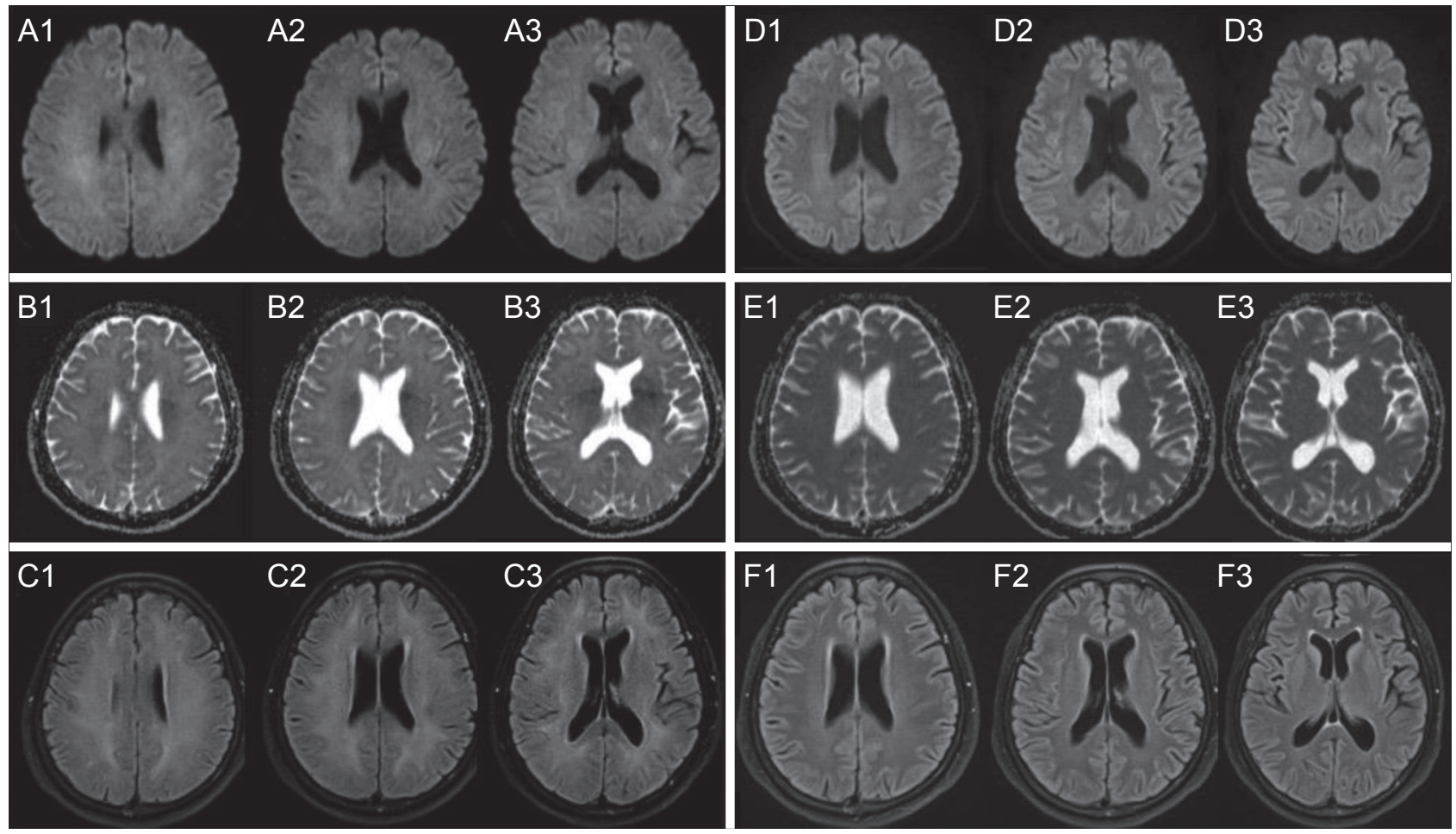

Figure 1. Brain MRI on day $13(A-C)$ and four months after presentation (D-F). Axial DWI $(A), A D C$ mapping (B) and FLAIR (C) images showing diffuse confluent high signal intensities on the bilateral cerebral white matter. Follow-up MRI (D-F) showing the disappearance of the previous abnormal signal intensities. MRI, magnetic resonance imaging; DWI, diffusion-weighted imaging; ADC, apparent diffusion coefficient; FLAIR, fluid-attenuated inversion recovery. 
level of protein, but other test results were unremarkable. The serum titers of anti-thyroglobulin (TG) antibody was increased as 1,175 IU/mL (normal, 0-115 IU/mL) and antithyroperoxidase (TPO) antibody was increased as $192.2 \mathrm{IU} / \mathrm{mL}$ (normal, 0-34 IU/mL). However, the level of TSH receptor antibody was normal as $1.63 \mathrm{IU} / \mathrm{L}$ (normal, 0-1.75 IU/L). The anti-neutrophil cytoplasmic antibody, anti-smooth muscle antibody, anti-nuclear antibody, and paraneoplastic antibody were all negative. Tumor screening tests were also negative. We diagnosed this patient as having $\mathrm{HE}$ and started intravenous methylprednisolone 1,000 mg/day for 5 days. On day 15, she was alert and oriented, and developed no further myoclonus or aphasic seizures. We started oral prednisolone $60 \mathrm{mg} /$ day for maintenance. The EEG was normal. On day 22, the serum titers of anti-TG, antiTPO, and TSH receptor antibody were decreased to 552.4 $\mathrm{IU} / \mathrm{mL}, 129.8 \mathrm{IU} / \mathrm{mL}$, and $1.02 \mathrm{IU} / \mathrm{L}$, respectively. The result of the thyroid function test was normal. Thus she was discharged with oral prednisolone $10 \mathrm{mg}$ per day. Four months after presentation, a follow-up MRI revealed the complete disappearance of the previous diffuse high signal intensities and she had no recurrence of symptom.

\section{DISCUSSION}

$\mathrm{HE}$ is a rare disease and the prevalence is approximately $2.1 / 100,000 .{ }^{4}$ It is a female-predominant condition, the ratio of men to women is $1: 5$, and most common age of onset is between 45 and $55 .{ }^{5}$ Most patients are affected by Hashimoto's thyroiditis, and some patients are affected by Grave's disease. ${ }^{6}$ There is no evidence that the anti-TPO antibody directly affects the central nervous system, and the exact pathogenic mechanism is still unknown. ${ }^{1}$

The clinical manifestations of $\mathrm{HE}$ are diverse, and may include epilepsy/disturbance of consciousness, cognitive impairment/memory loss, myoclonus hallucinations and psychotic symptoms, stroke-like symptoms, tremors and involuntary movements, language barriers, and ataxia. ${ }^{2}$ Our patient developed many clinical symptoms, such as stroke-like symptoms, tremors, myoclonus, status epilepti-
Cus, and a comatose mental status. The diagnostic criteria of HE includes 1) a lack of other diseases such as infection, stroke, metabolic diseases, and other factors accounting for the acute or subacute encephalopathy, 2) euthyroidism or thyroid hormone changes that are unable to justify the symptoms, 3) an association with autoimmune thyroid diseases with an elevated plasma anti-TPO antibody level, and 4) a favorable response to corticosteroid therapy. ${ }^{7}$ Our case was compatible with the diagnostic criteria.

There have been several reports of MRI findings in patients with HE, which showed high signal intensities on T2weighted and FLAIR imaging. The location and size of the lesions were variable. The reported locations of the lesions included the cortex, subcortex, brain stem, basal ganglia, hippocampus, splenium of corpus callosum, and the hemispheric white matter. ${ }^{8-10}$ However, MRI is unremarkable in most patients and there are no MRI features specific to the diagnosis of HE. ${ }^{10} \mathrm{MRI}$ abnormalities may be reversible with appropriate treatment. In our case, MRI showed diffuse confluent high signal intensities at the bilateral cerebral white matter on FLAIR imaging and ADC mapping. We considered the subtle high signals on DWI as a T2 shine through effect and lesions were exclusively confined to the white matter. Therefore, we interpreted this MRI findings as vasogenic edema and leukoencephalopathy.

The main first-line treatment for HE is methylprednisolone 1,000 mg as an intravenous infusion for 3-5 days. ${ }^{11}$ The symptoms usually respond within a week, sometimes as quickly as within a day. In up to $40 \%$ of patients, there is no recurrence after the first steroid pulse therapy. ${ }^{12}$ Our patient showed a positive response to methylprednisolone pulse therapy and maintenance therapy. For patients with a poor response to corticosteroids, use of the combination of corticosteroids and azathioprine, cyclophosphamide, plaquenil, methotrexate, intravenous immunoglobulin, or plasmapheresis have been reported. ${ }^{1}$

\section{Conflicts of interest}

The authors have no funding, financial relationships, or conflicts of interest to disclose. 


\section{REFERENCES}

1. Chang IS, Chang TC. Hashimoto's encephalopathy: report of three cases. J Formos Med Assoc 2014;113:862-6.

2. Tang Y, Xing Y, Lin MT, Zhang J, Jia J. Hashimoto's encephalopathy cases: Chinese experience. BMC neurol 2012;12:60.

3. Vernino S, Geschwind M, Boeve B. Autoimmune encephalopathies. Neurologist 2007;13:140-7.

4. Chong JY, Rowland LP, Utiger RD. Hashimoto encephalopathy: syndrome or myth? Arch Neuro/2003;60:164-71.

5. Ferracci F, Bertiato G, Moretto G. Hashimoto's encephalopathy: epidemiologic data and pathogenetic considerations. / Neurol Sci 2004;217:165-8.

6. Mocellin R, Walterfang M, Velakoulis D. Hashimoto's encephalopathy : epidemiology, pathogenesis and management. CNS Drugs 2007;21:799-811.

7. Chen N, Qin W, Wei C, Wang X, Li K. Time course of Hashi- moto's encephalopathy revealed by MRI: report of two cases. J Neurol Sci 2011;300:169-72.

8. Zhu Y, Yang H, Xiao F. Hashimoto's encephalopathy: a report of three cases and relevant literature reviews. Int / Clin Exp Med 2015;8:16817-26.

9. Song YM, Seo DW, Chang GY. MR findings in Hashimoto encephalopathy. AJNR Am J Neuroradio/ 2004;25:807-8.

10. Ramalho J, Castillo M. Hashimoto's encephalopathy. Radiol Case Rep 2015;6:445.

11. Pozo-Rosich P, Villoslada P, Canton A, Simo R, Rovira A, Montalban $X$. Reversible white matter alterations in encephalopathy associated with autoimmune thyroid disease. / Neurol 2002;249:1063-5.

12. Bohnen NI, Parnell KJ, Harper CM. Reversible MRI findings in a patient with Hashimoto's encephalopathy. Neurology 1997:49:246-7. 